\title{
Usos específicos de los blogs como herramienta educativa
}

\author{
Fernando Checa García \\ Euro-Mediterranean University Institute. \\ Universidad Complutense de Madrid
}

http://dx.doi.org/10.5209/rev_NOMA.2014.v42.n2.48773

Resumen.- En este artículo planteamos algunas de las opciones que pueden ofrecer los blogs y las bitácoras educativas como apoyo al proceso de enseñanza y aprendizaje. Tantos desde el punto de vista del profesor como desde el ámbito del estudiante, los blogs ofrecen amplias posibilidades que permiten conjugar el espacio de expresión así como la relación con el entorno global expresado en términos de internet. Los contenidos y los hiperenlaces, las relaciones entre los usuarios y las redes que se crean entre los participantes de la comunidad blog se plantean de esta manera como un complejo espacio de apoyo al crecimiento en el aprendizaje independiente y pertinente de estudiantes y profesores. Blogs como actividad de lectoescritura y blogs como desarrollo de la crítica de forma independiente.

Palabras Clave.- Blogs Educativos, Bitácoras, Redes Sociales, Tecnología Educativa

Abstract.- In this paper we present some of the options that can offer the educational blogs to support the teaching and learning process. From the point of view of the faculty as from the view of student blogs its offer big opportunities that allow conjugate the space of expression and the relationship with the global environment expressed in terms of Internet. The contents and hyperlinks, the relations between users and networks, are created between the participants of the blog community arise in this way as a complex space to support growth in the independent and relevant learning for students and teachers. Blogs work as literacy activity and as developing critical independently.

Keywords.- Educational Blogs, Blogs, Social Networks, Educational Technology

\section{Introducción}

Los blogs han sido estudiados como herramienta educativa desde hace ya varios años. Son herramientas que han sido incorporadas con bastante celeridad en los entornos educacionales debido a sus posibilidades de creación, participación y colaboración.

Podemos mostrar en una matriz los usos más importantes que pueden tener los blogs desde los dos planos, de lectoescritura y tanto desde el punto de vista del estudiante como desde la posición del profesor. Los actores de la formación que se entrecruzan en el proceso y los dos usos fundamentales. Debemos hacer hincapié en que antes de comenzar la edición de un blog es necesario lograr entender en qué consisten y convertirse en un lector de los mismos para poder interiorizar los diferentes estilos y retos que se plantean en su desarrollo. La curva de aprendizaje en el uso de blogs, especialmente desde el punto de vista de los docentes, no es especialmente complicada, pero sin duda es 
fundamental que exista un trabajo previo de acercamiento a estos entornos, muy fecundos dada su popularización cada vez mayor. Tan importante va a ser el trabajo en la edición y publicación de los posts como el acceso y la lectura de otros blogs (Levine, Lamb, D’Arcy, 2004).

Así pues, analizaremos los usos específicos desde el punto de vista de las dos actividades básicas, lectura y escritura, y desde la visión de estas vías de trabajo por parte de los docentes y de los alumnos.

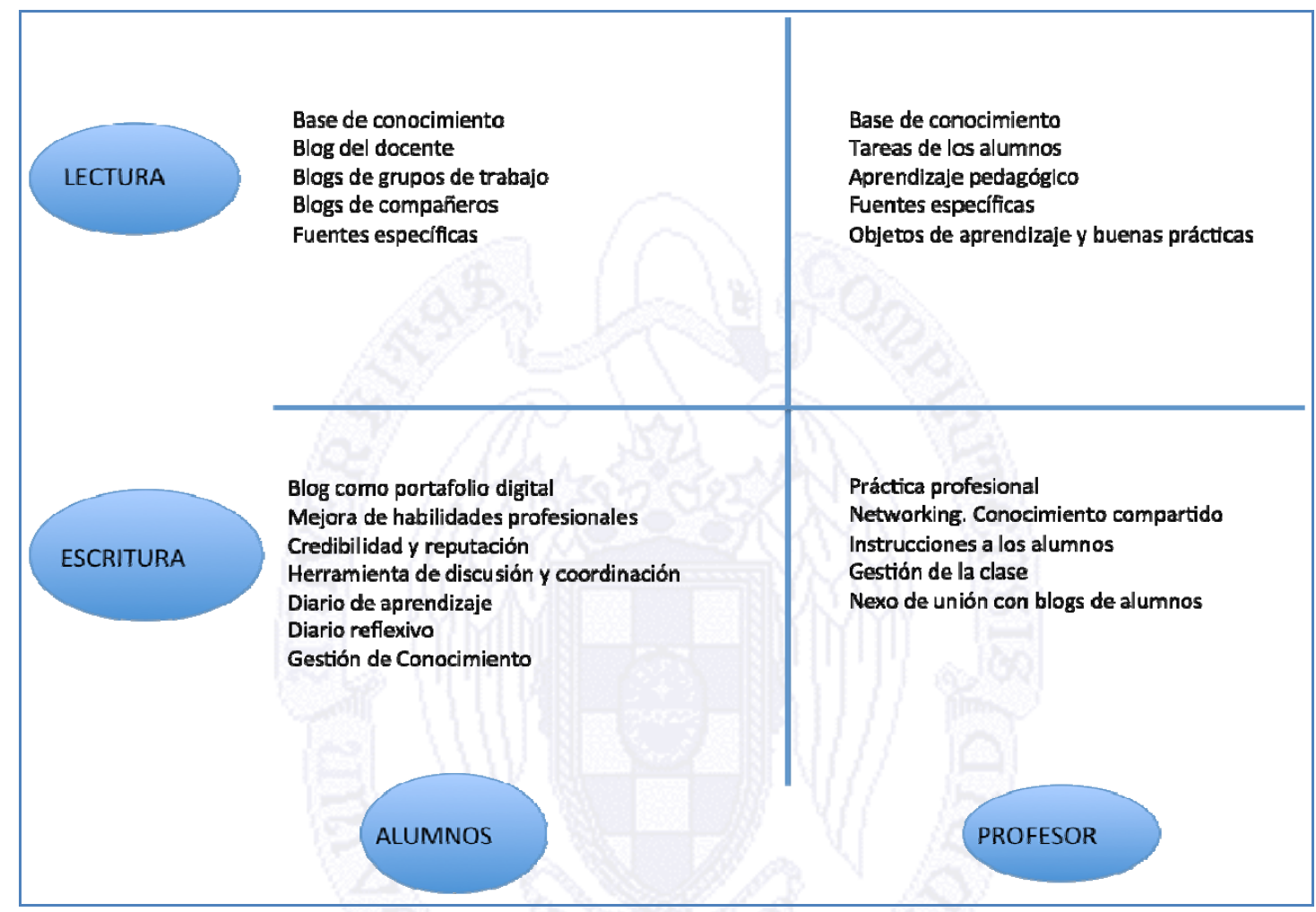

Figura 1. Algunos usos de los blogs en educación

Desde el punto de vista de la lectura de blogs estos cumplen distintas funciones que incrementan la capacidad de enseñanza y aprendizaje de los participantes, tanto alumnos como profesores. Veamos con detenimiento algunas de sus posibilidades:

\section{Lectura de blogs por parte de los estudiantes}

Los alumnos pueden acceder a nuevas bases de conocimiento. Incrementando la información obtenida desde las fuentes científicas y las publicaciones de referencia de la materia, el reconocimiento de los materiales que se están creando en otros centros educativos, así como el seguimiento y la creación de una base de conocimiento a la que acudir a lo largo de su vida académica, permite que el alumno observe como el conocimiento se va desarrollando y evolucionando. 
La lectura del blog del profesor es el complemento a las sesiones en las que el docente expone partes de la materia y genera discusiones o actividades a realizar. El acceso frecuente a la publicación del profesor permite al alumno ver las evoluciones de su facilitador y superar los posibles frenos o miedos en la edición del suyo, además de poder interactuar a través de los comentarios y obtener una fuente de contenido extra para alimentar su propio blog.

La mayoría de las plataformas que ofrecen un servicio de blogs permiten la posibilidad de crear blogs grupales. Para la realización de trabajos colaborativos el blog grupal se muestra como una herramienta excelente que ayuda a los miembros del grupo de trabajo interactuar, publicar sus conclusiones identificadas de forma individual y generar un reparto de tareas en la gestión del blog. La lectura del blog grupal permite al alumno interiorizar mejor los conceptos que plantean sus compañeros de grupo, enfrentarse a ellos en un plano de igualdad, aumentar su sentido crítico y apoya la colaboración para alcanzar objetivos comunes.

No solamente el blog del grupo ha de convertirse en una lectura constante sino el del resto de compañeros. Las nuevas ideas generadas por otros miembros de la clase o los avances en aspectos como el diseño o la introducción de temas inicialmente no tratados en las asignaturas puede servir de inspiración así como incrementar los lazos sociales y el conocimiento más allá de los grupos que tradicionalmente se forman en función de las afinidades conocidas.

Para que los alumnos puedan mejorar sus habilidades en la lectura de blogs es necesario que se desmitifique que la información aparecida en ellos tiene un carácter estrictamente amateur. Saber encontrar las fuentes de información, utilizar activamente los buscadores para encontrar nuevas fuentes de información, como el propio Google, Blogsearch o Bitácoras ayuda a que los alumnos incrementen el sentido de la crítica sobre los contenidos que se publican. Y para poder hacer una gestión adecuada de las fuentes el uso de lectores de feeds que ayuden a gestionar la gran cantidad de contenidos es básico. La figura del profesor como ayuda para discernir entre las distintas fuentes y como facilitador en la elección y el uso de los agregadores de fuentes RSS aparece en un plano protagonista. El alumno debe tener la libertad de elegir la aplicación de gestión con la que se encuentre más cómodo y para ello debe conocer las más comunes, sus características y funcionalidades, sus puntos de debilidad frente a sus competidoras. Los sencillos usos de un lector de feeds no deben darse por supuestos y es por ello que la formación en conceptos como la sindicación de contenidos, el filtrado de fuentes o la búsqueda activa ha de convertirse en una de las primeras etapas del trabajo.

\section{La lectura de blogs por parte del profesor}

Al igual que los alumnos pueden acceder a bases de conocimiento gracias a la lectura de blogs, el profesorado tiene en esa posibilidad un camino para incrementar su acceso a unos contenidos que, si bien pueden no tener las características aplicables al conocimiento científico publicado en revistas o papers de calidad reconocida, no debe ser desdeñable. Muchos de los avances que posteriormente son publicados en revistas o libros con índice de impacto 
son previamente avanzados en blogs de investigadores. Cada vez es más frecuente que profesionales y directivos lleven adelante un blog en el que muestran los avances de su sector, de su empresa o sus reflexiones sobre cualquier campo de actividad. El conocimiento compartido puesto a disposición a través de miles de blogs y de acceso libre y sencillos de encontrar debe convertirse en una herramienta de consulta permanente por parte del docente universitario de forma que su vertiente necesaria de investigador no se vea descuidada y el acceso a las nuevas publicaciones así como a las fuentes específicas de su campo de estudio y trabajo lleguen a él de forma fluida y permanente.

El profesor ha de leer los blogs de sus alumnos. Pedir que los estudiantes escriban en un blog para luego no acceder al mismo, valorar sus posts, criticar aquellas aseveraciones que puedan no ser correctas o practicar una lectura asertiva, significa que la incorporación de los blogs en la dinámica de la clase se vea abocada al fracaso. El primer lector del blog de los estudiantes ha de ser el profesor y su lectura debe hacerse notar. De ahí la importancia de la participación activa en los comentarios empujando y generando debate, ofreciendo su ayuda e introduciendo los contenidos leídos en la clase. De nuevo se convierte en fundamental la organización de los blogs utilizando un lector de feeds donde aparezcan claramente definidos los blogs de sus alumnos así como aquellos posts que deban ser utilizados en el aula para su comentario o trabajo sobre ellos.

El tiempo repartido entre la docencia y la investigación de los profesores universitarios españoles suele dejar poco espacio para la formación en nuevas técnicas pedagógicas y en avances en las metodologías docentes. Las universidades intentan ofrecer a sus profesores cursos de reciclaje pero en muchas ocasiones estos se solapan con la actividad diaria, resultando poco motivantes para un docente que puede encontrar en ellos un incremento extra en su carga de trabajo. Por ello mediante la lectura de blogs especializados en innovación docente y metodologías educativas el profesor puede estar al tanto con facilidad de los avances que otros compañeros están llevando a cabo. Las islas de conocimiento que a veces se producen en los entornos educativos por parte de profesores que llevan adelante innovaciones y no son conocidas por el resto de la comunidad educativa, tienen su contrapartida en el acceso a las experiencias y buenas prácticas de profesores que editan blogs, en cualquier parte del mundo y sobre cualquier temática.

Poder acceder a nuevos objetos de aprendizaje, compartidos por profesores desde su blog, permite enriquecer los contenidos propuestos a los alumnos. Evita un tiempo precioso en la redacción y creación de materiales ya realizados y puestos a disposición de la comunidad bajo licencias Creative Commons. Tradicionalmente el profesor crea y reutiliza sus objetos de aprendizaje repitiendo pautas ya puestas en marcha por otros compañeros. Las clásicas transparencias que acompañan a la docencia de contenidos de un tema suelen ser creadas de forma individual, cuando a través de la lectura de blogs es factible acceder a materiales compartidos en repositorios como Slideshare. De igual forma que se anima al estudiante a que utilice materiales creados por terceros manteniendo siempre la cita al autor original, el profesor puede 
servirse de esos materiales sin que su prestigio tenga por qué verse menoscabado.

\section{La escritura de blogs por parte de los estudiantes}

El blog del alumno es una excelente forma de incorporar a sus asignaturas su portafolio digitalizado, en el que se van añadiendo las tareas y trabajos solicitados para alcanzar los objetivos de aprendizaje previamente conocidos. Frente a las tradicionales entregas de prácticas al profesorado, de forma escrita o a través de medios digitales como los campus virtuales o el correo electrónico, la creación y mantenimiento de un portafolio online incide en la construcción de la identidad online y en la responsabilidad ante la calidad y el esfuerzo puesto en los trabajos llevados a cabo. Enfrentarse a la indagación de sus compañeros y de la comunidad de Internet, de forma abierta y sin barreras, lleva a que el estudiante mejore sus habilidades comunicativas y de redacción, así como su capacidad de síntesis y de reflexión personal. La consciencia de que su obra pueda ser conocida por cualquiera genera tensión y resquemores al comienzo, pero una vez superados se revierte en motor de aprendizaje.

Enfrentarse al aprendizaje conseguido desde el plano de la reflexión permanente es otro de los aspectos novedosos que incorpora el uso de los blogs. Inicialmente el alumno acude a clase, estudia o trabaja sobre unos contenidos y finalmente obtiene una calificación tras ser evaluado, generalmente bajo aspectos objetivos y subjetivos por parte del profesor, garante de la consecución de los objetivos de aprendizaje. Pero la reflexión sobre el aprendizaje obtenido no solía plantearse hasta hace pocos años. Si el alumno obtenía una calificación que le permitiera superar los requerimientos mínimos se consideraba que había logrado ese aprendizaje, sin que su opinión fuera más allá que el reflejo ocasional en encuestas de satisfacción con la asignatura. Con la puesta en marcha de un blog y la motivación por el profesorado para que el alumno escriba sobre ello sin temor, es posible saber qué es lo que considera el alumno que ha aprendido. Los objetivos de aprendizaje suelen estar marcados al comienzo del curso por el profesor de acuerdo con las características curriculares de la materia y no suelen ser pactados ni tan siquiera discutidos con los alumnos. El planteamiento vertical es generalizado. La reflexión pública sobre el día a día y el grado de alcance de los objetivos da, por el contrario, una información excepcional al profesor que puede ser consciente de errores en el planteamiento de la materia. El estudiante cuando se siente libre para la reflexión en público se enfrenta consigo mismo y aprende a valorar o criticar a través de sus sentimientos y palabras el valor o la falta del mismo de lo aprendido.

La crítica a su aprendizaje y la puesta en público de su trabajo incide en el crecimiento de la credibilidad y en el aumento de la reputación adquirida y reflejada en el momento en que el estudiante se ve a si mismo como parte creadora de los contenidos de Internet, referenciado por otros, comentado y envuelto en conversaciones sobre desarrollo académico. Ya no es un estudiante que gestiona su imagen en público o digitalmente entre su círculo de amigos. Se convierte en un individuo con algo que decir, que puede ser escuchado y valorado no sólo por el docente sino por cualquiera que acceda a 
su blog. La reputación adquirida a lo largo del tiempo que el blog permanezca en activo y su interrelación con otras herramientas de la Web 2.0, como las redes sociales profesionales, pueden situar al estudiante en una posición de privilegio tras su recorrido académico y su inserción profesional.

La entrada en la conversación, la participación con comentarios en los blogs de otros compañeros y en el del profesor, la incorporación de las críticas y de las sugerencias en su discurso, la ordenación mediante categorías y uso de etiquetas semánticas convierten, pues, al blog del alumno en una excelente herramienta de carácter global con objetivos específicos susceptible de servir para gestionar su conocimiento, para habituarse a transferir y compartir, a trabajar en un entorno de colaboración y a que la participación activa en Internet genere valor, tanto para sí mismo como para el resto de la comunidad.

\section{La escritura de blogs por parte del profesor}

Los profesores universitarios tienen dos vertientes fundamentales en su trabajo, la investigadora, que les convierte en creadores de conocimiento, en indagadores de su ámbito de estudio y transmisores de sus logros investigadores al resto de la comunidad científica así como al resto de la sociedad. Así mismo sus investigaciones revierten en sus capacidades docentes, pudiendo mostrar el camino a seguir a los alumnos, facilitándoles los recursos más interesantes y orientándoles en el proceso de construcción de su aprendizaje, tanto desde el punto de vista de los contenidos como desde las competencias profesionales requeridas para la inserción laboral. Muchos profesores también compatibilizan estas dos labores con las propias de la práctica profesional de su campo, incorporando a su vez a su conocimiento la realidad tangible de la puesta en funcionamiento de sus enseñanzas. Poder llevar adelante un blog supone incrementar la visibilidad de su práctica profesional. Compartir los avances en sus investigaciones previamente a la redacción de un paper o libro con los lectores y los miembros de la comunidad de Internet puede ser un buen ejercicio de reflexión previo a la redacción del trabajo definitivo. Poner disponibles en el blog los trabajos ayuda a darles visibilidad, más allá de la publicación en revistas muchas veces de difícil acceso para personas ajenas a la comunidad universitaria, pero con intereses sobre el tema. Compartir objetos de aprendizaje y buenas prácticas incrementa el prestigio profesional y sitúa al profesor como experto reconocible no solo dentro de su universidad, sino fuera de ella.

La visibilidad que ofrecen los blogs ayuda también a que los profesores que escriben en ellos obtengan un incremento en su red de contactos con profesores y expertos de otras universidades y áreas de estudio. De ese contacto pueden salir colaboraciones para la realización de trabajos conjuntos y nuevos proyectos que mejoren la posición profesional del profesor. La identidad y visibilidad digital de cualquier usuario de Internet se muestra especialmente importante en profesionales dedicados a la docencia y la investigación. No podemos olvidar que el profesorado tiene siempre un perfil de servicio público y que cada vez más sujetos cercanos a su entorno van a poder interesarse por su trabajo y sus inquietudes. La edición frecuente de un blog y 
la repercusión que esta acaba teniendo gracias a los motores de búsqueda, convierte al profesor en un experto tanto dentro como fuera de su universidad.

El blog del profesor ha de convertirse en el espacio al que los alumnos acuden de manera reiterada para obtener instrucciones sobre cuestiones relacionadas con la asignatura, donde pueden publicarse los materiales utilizados en clase así como los recursos de apoyo de cualquier tipo. El blogroll del profesor es el mejor espacio para realizar recomendaciones acerca de sitios de interés y fuentes de necesaria consulta. La capacidad de integración de vídeo y audio ayuda a que los suscriptores, alumnos o no, puedan seguir ejercicios de forma diferente a lo habitual (e.j. un profesor puede grabar un pequeño podcast semanal en el que sitúe algunos de los puntos que han de tratarse en la siguiente clase sin demasiado esfuerzo). La propia dinámica de las clases, de igual forma que el alumno sigue su diario reflexivo de aprendizaje, puede ser publicada para mostrar a los alumnos que la materia es construida de forma conjunta entre todos los miembros de la clase, incluyendo al profesor, sin menoscabo de la autoridad moral de su figura como facilitador del conocimiento. El blog del profesor permite realizar una gestión de las sesiones y de los avances obtenidos, ofreciendo los resúmenes y reflexiones finales sobre las distintas unidades de conocimiento.

Por último, el blog del profesor puede ser también el hub desde el que se enlazan todos los blogs de los alumnos. Especialmente en clases con un gran número de estudiantes resulta interesante que haya un sitio en el que se encuentren referenciados todos, para que cualquier miembro pueda volver sobre ellos y poder encontrar con facilidad el blog de sus compañeros.

Si la ordenación de los posts a través de categorías y mediante etiquetas es siempre importante, lo es especialmente en el caso del blog del profesor. Los estudiantes que lo van a visitar continuamente a lo largo del curso han de tener la posibilidad de acceder a todos los contenidos sin dificultades y de la forma más sencilla posible. La ordenación de los posts se convierte de esta forma en algo fundamental para la correcta gestión del conocimiento allí reflejado. Casi tanto como el estilo de redacción que ha de ser cálido y cercano sin que ello signifique dejar de lado la rigurosidad de los temas allí tratados y comentados.

Un último aspecto que el profesor ha de tener en cuenta en el momento de gestionar su asignatura con un blog es el espacio tiempo. Las asignaturas tienen una duración determinada y habitualmente se imparten de nuevo al año siguiente a otro grupo. Ante esa situación común pueden plantearse dos escenarios: la creación de un blog por cada asignatura impartida y por cada curso académico permite ir acompasado con la escritura de los estudiantes que ven como el profesor va escribiendo casi al mismo tiempo que ellos. Cuando el alumno observa el blog del profesor y ve decenas de posts puede tender a sentir cierta presión ante la gran cantidad de contenidos. Sin embargo si el alumno observa como el profesor va escribiendo su blog al mismo tiempo que él mismo y el resto de sus compañeros la situación se torna en motivante. Pero por otro lado, el hecho de iniciar un blog cada vez que se comienza una asignatura, abandonando el anterior, puede llevar a no reutilizar objetos de aprendizaje interesantes. Habrá de ser el profesor el que decida, según su habilidad técnica, la herramienta blog que utilice y sus intereses en el 
planteamiento de la materia la reutilización de un blog año tras año o la apertura de uno nuevo por asignatura. En el caso de reutilizar el mismo blog, la personalización de los post cada inicio de curso, así como la categorización de los contenidos, no solo por temas, sino por grupos, ha de resultar muy clara. Los blogs de asignatura pueden acompañarse con la edición del blog personal del profesor, en el que se publica no solo sobre el día a día de la clase, sino sobre los intereses particulares y cuya incidencia en los aspectos anteriormente reseñados acompañan e incrementan las posibilidades de desarrollo del profesor.

\section{Conclusiones}

Como hemos observado a lo largo de este artículo las posibilidades educativas que ofrecen los blogs son elevadas. Tanto desde el punto de vista de la lectura de contenidos como desde el propio espacio de la expresión escrita, los blogs se conforman como una de las herramientas que mejor se adecúan a la introducción de la tecnología en el aula. Sus fuertes capacidades para el desarrollo de tareas y la exposición que suponen hacen que deban ser tenidos en cuenta cada vez más por la comunidad académica. La incorporación de los blogs en el aula ha de contar, eso si, con el trabajo de profesores y alumnos, no quedando como parte exclusiva de trabajo de estos últimos. El profesor adquiere en este espacio una función fundamental como facilitador y apoyo en el uso y participación así como en gestor de los nuevos retos ante la evaluación que se generan con esta herramienta.

\section{Referencias bibliográficas}

BERG, I., ADMIRAAL, W. y PILOT, A., (2006); Designing student peer assessment in higher education: analysis of written and oral peer feedback. Teaching in Higher Education, Volumen 11(2), 135-147.

BLANCO, S., (2005); Los weblogs como herramienta didáctica en el seno de una asignatura curricular, El ecosistema digital: modelos de comunicación, nuevos medios y público en Internet, València, Servei de Publicacions de la Universitat de València, 151-166

CASTAÑEDA, L., (2007); Software Social para la escuela 2-0: más allá de los Bogs y las Wikis, Inclusión Digital en la educación superior: desafíos y oportunidades en la sociedad de la información, $X$ Congreso Internacional EDUTEC, Buenos Aires

CASTAÑO GARRIDO, C. y PALACIO, G., (2006); Edublogs para el aprendizaje contínuo en la Web Semántica, E-Actividades, un referente básico para la formación en Internet, 95-111, Sevilla: Mad,

CASTAÑO GARRIDO, C., (2008); Prácticas educativas en entornos Web 2.0, Madrid: Síntesis

CEREZO, J.M. (2206); La blogosfera hispana: pioneros de la cultura digital, Madrid: Fundación France Telecom

CHAMORRO, R., (2008); Blogs. Madrid: Creaciones Copyright 
CHECA, F., JOYANES, L., (2009); El uso de los blogs como metodología activa de aprendizaje: Innovando en Gestión del Conocimiento en VI Jornadas Internacionales de Innovación Universitaria. Universidad Europea de Madrid CHECA, F. (2009); The use of blogs as key element in the new TeachingLearning process" en Nomads, Mediterranean Perspectives, $\mathrm{n}^{\circ} 2$, , MadridMéxico: Paza \& Valdés

CHECA, F. (2013); La utilización del microblogging y de twitter como herramienta de enseñanza-aprendizaje en Espiral, Cuadernos del Profesorado, Vol. 6(11)

COSTA, C., BEHAM, G. y REHINHARDT, W. (2008); Microblogging In Technology Enhanced Learning: A Use-Case Inspection of PPE Summer School 2008 en Workshop at the European Conference on Technology Enhanced Learning (ECTEL), Maastricht

GARCÍA AGUILERA, F.J. y AGUILAR CUENCA, D. (2002); Ingeniería de la formación: cómo diseñar acciones de formación presenciales en base a criterios de calidad pedagógica, Capital humano: revista para la integración y desarrollo de los recursos humanos, Año número 15, Número 152, 40-47

GARCÍA AGUILERA et Al, (2004). Metodología didáctica aplicada: estrategias de intervención pedagógica, Málaga: Lavante

GONZÁLEZ SERNA, J. L., (2003); Weblog y Enseñanza, Perspectiva CEP. Revista de los centros del profesorado de Andalucía, Número 6, Noviembre JIMÉNEZ, R. y POLO, F., (2007); La Gran Guía de los Blogs. Barcelona: Elcobre Ediciones

LARA, T., (2009); El papel de la Universidad en la construcción de su identidad digital. En: "Cultura digital y prácticas creativas en educación" [monográfico en línea]. Revista de Universidad y Sociedad del Conocimiento (RUSC). Vol. 6(1) UOC.

LEVINE, A, LAMB, B, D'ARCY, N, (2004) "Connecting Learning Objects with RSS, Trackback, and Weblogs", NMC conference, 2003, disponible en línea en http://www.mcli.dist.maricopa.edu/show/nmc1003/

MARTí, D., (2008); Blogs educativos de comunicación en Iberoamérica, Revista Diálogos de la Comunicación, Número 76, Enero-Junio.

MARZAL, M. A., y BUTERA, M. J., (2007); Los blogs en el nuevo modelo educativo universitario: posibilidades e iniciativas, BiD, Textos universitaris de biblioteconomia i documentació, Facultad de Biblioteconomia y Documentación, Universitad de Barcelona, número 19. 